\title{
Giemsa Staining of Mitotic Chromosomes in Kluyveromyces lactis and Saccharomyces cerevisiae
}

\author{
By CESIRA L. GALEOTTI AND KEITH L. WILLIAMS \\ Genetics Department, Research School of Biological Sciences, The Australian National \\ University, P.O. Box 475, Canberra City, A.C.T. 2601, Australia
}

(Received 25 July 1977; revised I4 September 1977)

\section{INTRODUCTION}

Visualization of yeast chromosomes by light microscopy of whole cells has so far been unsatisfactory (McCully \& Robinow, 1971). Although the use of protoplasts has improved preparations, it has still not proved possible to visualize metaphase chromosomes with the light microscope (Fischer, Binder \& Wintersberger, 1975). Extensive comparative studies with light and electron microscopy on yeast mitotic chromosomes have also failed to reveal metaphase chromosomes, hence chromosome condensation and arrangement on a metaphase plate in yeast has been postulated to differ from the respective processes in higher organisms (McCully \& Robinow, I97I). Nevertheless, a rapid and simple method for the assessment of chromosome numbers in yeast species would be most useful for taxonomic studies and where hybridization between different species and genera is contemplated. Previous success with air-drying and Giemsa-staining techniques for cytological examination of a simple eukaryote Dictyostelium discoideum (Brody \& Williams, I974; Robson \& Williams, 1977) suggested investigation of this method for the examination and possibly for the identification of yeast chromosomes.

Hybrids are being constructed between Kluyveromyces lactis and Saccharomyces cerevisiae. This report details studies on the chromosomes of these species which were undertaken to provide a cytological basis, in addition to genetic markers, for differentiating the two species and their hybrids.

\section{METHODS}

Preparation of protoplasts. Haploid strains of Kluyveromyces lactis (laboratory strain ERY ${ }^{\mathrm{R}} 25$ ) and Saccharomyces cerevisiae (ATCC26500) were used. Actively growing cultures (about $3 \times 10^{7} \mathrm{cells} \mathrm{ml}^{-1}$ ) in GYP medium $\left[0.5 \%(\mathrm{w} / \mathrm{v})\right.$ yeast extract, $\mathrm{I} \cdot 0 \%(\mathrm{w} / \mathrm{v})$ peptone, $2 \%(\mathrm{w} / \mathrm{v})$ glucose] at $30{ }^{\circ} \mathrm{C}$ were washed twice with water. Washed cells were resuspended to a final density of about $10^{8}$ cells $\mathrm{ml}^{-1}$ in citrate/phosphate buffer ( $\mathrm{pH} 5.4 ; 0.2 \mathrm{M}-\mathrm{Na}_{2} \mathrm{HPO}_{4}$. I $2 \mathrm{H}_{2} \mathrm{O}$, O.I M-citric acid. $\mathrm{H}_{2} \mathrm{O}$ ) containing $0.6 \mathrm{M}-\mathrm{KCl}$ as osmotic stabilizer. Driselase (Kyowa Hakko Kogyo Co., Tokyo, Japan) and snail gut juice (Industrie Biologique Francaise, 921 I 5, Clichy, France) were added to final concentrations of $2 \%(\mathrm{w} / \mathrm{v})$ and $0.5 \%(\mathrm{v} / \mathrm{v})$ respectively to the washed cells, which were allowed to stand at $30^{\circ} \mathrm{C}$ for about $30 \mathrm{~min}$ in the case of $K$. lactis and $60 \mathrm{~min}$ for $S$. cerevisiae. This procedure for producing protoplasts was carried out directly in $10 \mathrm{ml}$ graduated centrifuge tubes.

Fixation and staining. Protoplasts were harvested by centrifuging at $150 \mathrm{~g}$ for $10 \mathrm{~min}$ and the pellet was fixed immediately (without any washing) in methanol/acetic acid $(3: \mathrm{I}, \mathrm{v} / \mathrm{v})$ as described previously (Brody \& Williams, 1974) except that the centrifugation was extended to $10 \mathrm{~min}$ at each step. The protoplasts were air-dried on to clean slides and stained with $10 \%$ (v/v) Gurr's Giemsa stain (Improved R66) in $0.067 \mathrm{M}$ Sorensen's phosphate buffer ( $\mathrm{pH} \mathrm{6.8)}$ for $40 \mathrm{~min}$. Usually slides were treated with $0.25 \%(\mathrm{w} / \mathrm{v})$ trypsin (Difco, $\mathrm{I}: 250$ ) in $0.85 \%(\mathrm{w} / \mathrm{v}) \mathrm{NaCl}$ for $5 \mathrm{~min}$ immediately before Giemsa staining.

Microscopy and photographic techniques. Slides were examined with a Carl Zeiss photomicroscope using bright field optics (plan apo 100/I 3 objective) and a green filter. Some photographs were taken with Kodak Panatomic X film (Robson \& Williams, 1977) but Agfa Copex Pan rapid film was found to be more suitable and was used in most experiments. The Agfa film was developed with Agfa Rodinal I:50 (v/v) for 7 min at $20^{\circ} \mathrm{C}$. 


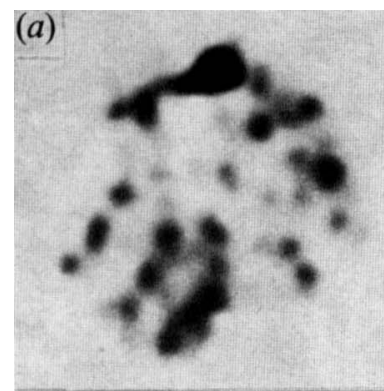

\section{(b)}
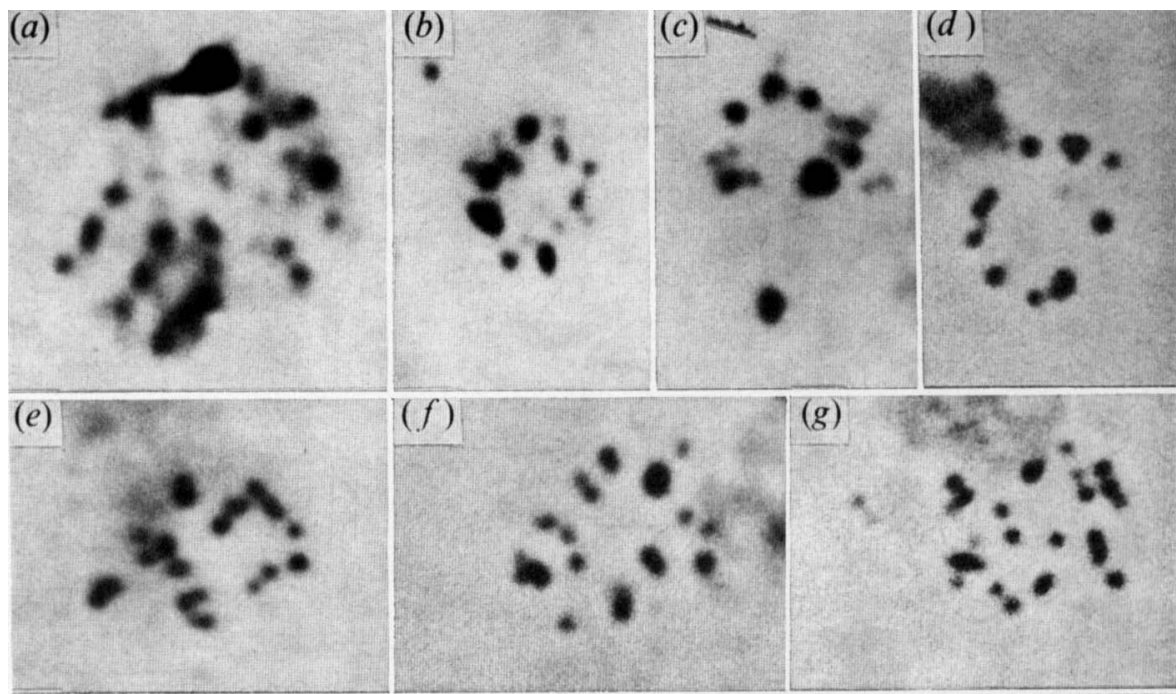

(g)

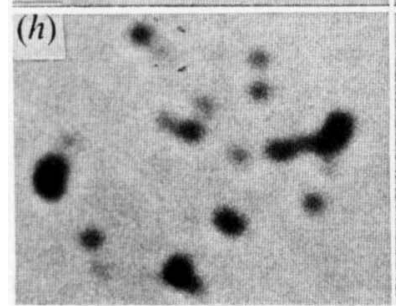

(i)

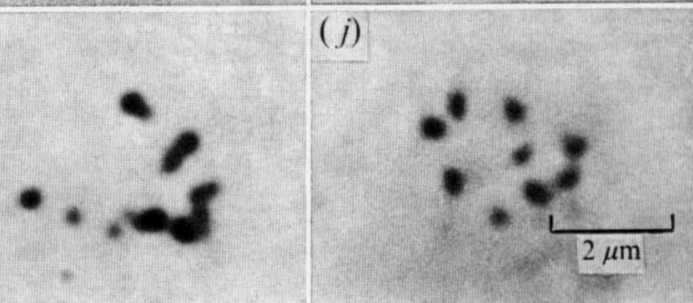

(k)

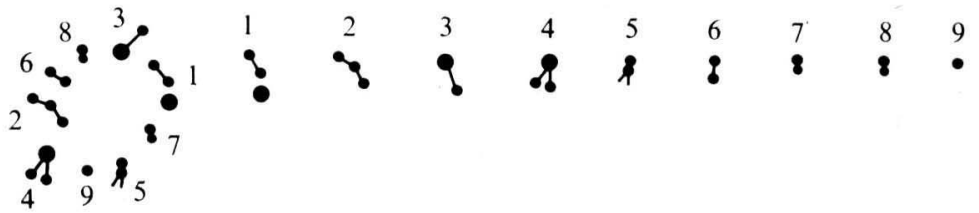

Fig. I. Mitotic chromosomes of $K$. lactis. Progressive condensation of chromosomes on to the metaphase plate $(a-d)$, well-spread metaphase chromosomes $(e-h)$ and apparent connections between chromosomes $(i-j)$. All photographs are at the same magnification; the scale is shown in $(j)$. An interpretative drawing of $(f)$ and numbering of the chromosomes is shown in $(k)$.

Comments on the method. The aim of this work was to develop a rapid and reliable technique for examining yeast chromosomes. While the procedure outlined involves the use of protoplasts, it was found preferable to use a crude fast technique rather than to emphasize high protoplast yield. Incubating protoplasts for various times in GYP medium plus $0.6 \mathrm{M}-\mathrm{KCl}$ before fixation was tried, but freshly prepared protoplasts gave the most satisfactory results. Furthermore the best preparations were obtained when protoplasts were harvested from the protoplasting solution and fixed without washing.

Trypsin treatment was useful for lightening the cytoplasmic staining in both species and often resulted in some differential staining along the chromosomes. Variability in staining characteristics is observed with Giemsa and often only a small portion of the protoplasts near the edge of the slide were suitable for examining chromosomes. 


\section{RESULTS}

\section{Mitotic chromosomes of $K$. lactis}

Chromosomes of $K$. lactis at different stages of condensation are shown in Fig. I $(a-d)$. Early prophase chromosomes (not shown) are barely discernible and appear as a tangled mess. As they condense, separate chromosomes can be discerned but their banded unwound appearance makes any attempt at counting very difficult (Fig. I $a$ ). After further condensation, chromosomes are disposed in a ring resembling a typical metaphase plate and approximately nine chromosomes can be counted (Fig. I $b-d$ ). Several representative wellspread metaphases are shown in Fig. I $(e-h)$. The trypsin treatment causes 'banding' so that individual chromosomes have a characteristic appearance. In Fig. $\mathrm{I}(k)$ an interpretative drawing of Fig. I $(f)$ is given which suggests that nine chromosomes with characteristic morphologies can be discerned. While the morphology and number of bands on each chromosome varies with the degree of contraction of the chromosome (e.g. compare Fig. I $a$ and $c$ ), there are similarities observed in different metaphases. On the basis of the numbering given in Fig. I $(k)$ most chromosomes in Fig. I $(e)$ and Fig. I $(h)$ can be recognized. Connections between chromosomes were also observed (Fig. $\mathrm{i}, \boldsymbol{j}$ ), sometimes involving several chromosomes (probably five connected end to end in Fig. $\mathrm{I} i$ ). Nevertheless where reliable counts can be made the number of chromosomes in haploid $K$. lactis is $9 \pm \mathrm{I}$.

\section{Mitotic chromosomes of $S$. cerevisiae}

Associations between chromosomes were frequently observed in $S$. cerevisiae either as densely staining groups (Fig. $2 a, b$ ) or chromosomes joined end to end (Fig. 2g). The reasons for this clumping are unknown. Since an estimate of the number of chromosomes in S. cerevisiae is already available from genetic studies (Mortimer \& Hawthorne, 1969) and cytogenetic observations (Tamaki, 1965; Wintersberger, Binder \& Fischer, 1975; Byers \& Goetsch, I975) only a limited number of preparations were examined. The haploid chromosome number deducible from these preparations is at least 15 (Fig. $2 c-h$ ), in general agreement with the above mentioned studies. There were morphological differences between chromosomes which, with further study, may allow identification of at least some of them.

\section{DISCUSSION}

Early studies on fungal cytology suggested that many features of the chromosomes and the mitotic cycle were unlike those of more complex eukaryotes. It has become clear that at least some of these apparently unusual features resulted from difficulties with the experimental techniques and were not inherent in the organisms (e.g. yeast histones are now thought to be similar to those of higher organisms: Wintersberger, Smith \& Letnansky, 1973; Franco, Johns \& Navlet, 1974). McCully \& Robinow (1971) showed that Schizosaccharomyces pombe had a mitotic spindle but still concluded that the chromosomes were not arranged on a metaphase plate. Our observations for $K$. lactis suggest that a metaphase plate is formed in this yeast. The technique we outline should allow increased resolution of chromosomal behaviour and the mitotic cycle not only in yeasts but also in other fungi.

The purpose of this paper was to see if chromosome numbers of different yeasts could be counted using a simple staining technique and light microscopy. The chromosomes are very small, on the limit of resolution of the light microscope, but used with care the method outlined can give chromosome numbers. Association between chromosomes causes problems, especially with $S$. cerevisiae, as has been observed by others (Wintersberger et al., 1975). However by examining less-condensed preparations, individual chromosomes can be seen. A problem with Giemsa staining is that chromosomes do not stain uniformly and this is enhanced by trypsin treatment. On the positive side this differential staining allows the identification of chromosomes and a tentative karyotype for $K$. lactis is proposed. 
(a)

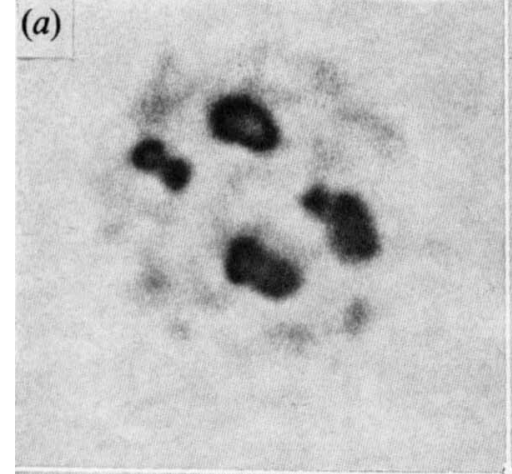

(c)

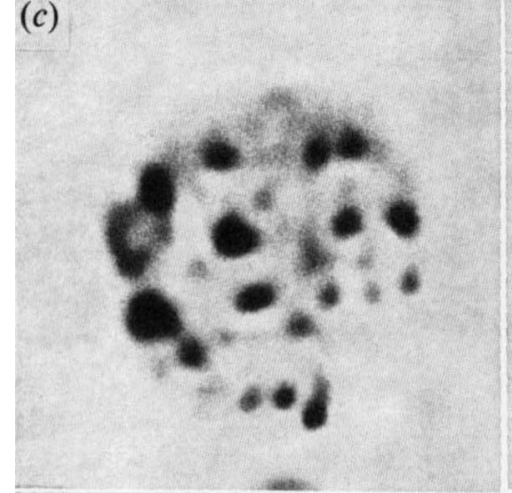

(e)

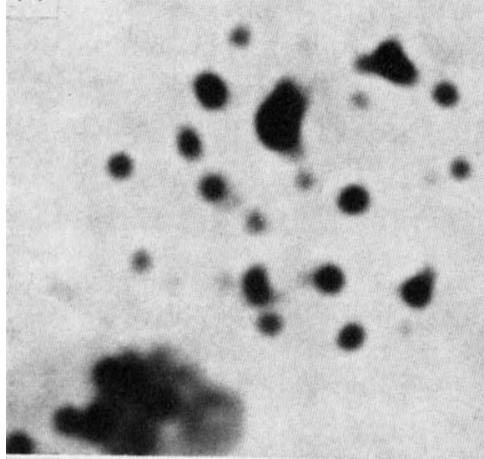

(g)

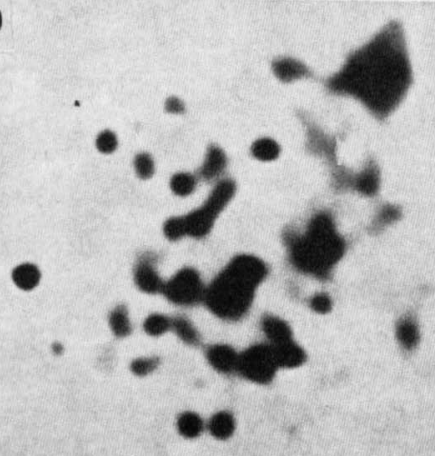

(b)

(d)

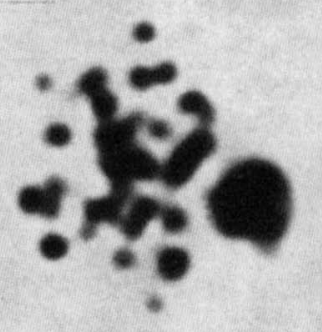

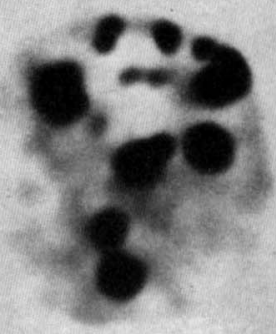

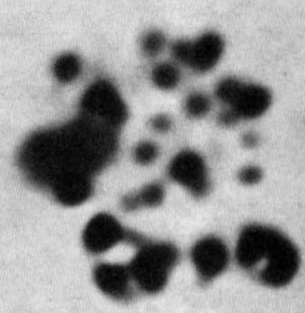

(f)

(h)
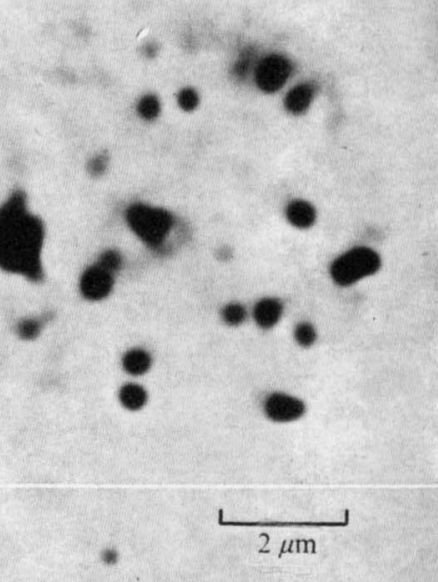

\&
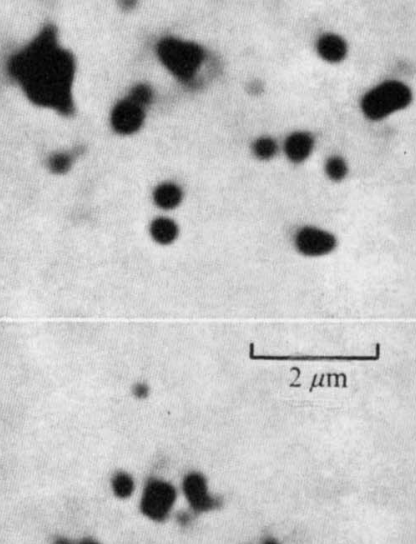

the

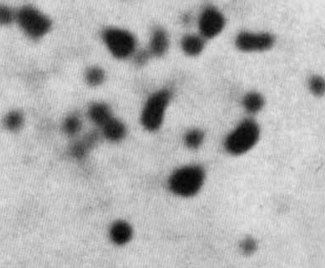

Fig. 2. Mitotic chromosomes of $S$. cerevisiae. $(a, b, g)$ Associations between chromosomes; $(c-f, h)$ well-spread metaphase chromosomes. All photographs are at the same magnification; the scale is shown in $(h)$. 
Knowledge of chromosome number provides additional information for deciding about taxonomic status. This may be particularly valuable in yeasts where morphological features are few. An example is the controversy concerning the classification of Kluyveromyces lactis. Campbell (1972) proposed that the genus Kluyveromyces should be reincluded in the genus Saccharomyces. Evidence on DNA homology suggests that $K$. lactis and $S$. cerevisiae are unrelated (Bicknell \& Douglas, 1970). The difference in chromosome number observed in this study and predicted previously on the basis of genetic evidence (Tingle, Herman \& Halvorson, 1968) would support the separation into two genera.

We thank Dr Graham Webb for advice on photographic techniques and Dr D. G. Clark-Walker and Professor M. White for helpful comments.

\section{REFERENCES}

Bicknell, J. N. \& Douglas, H. C. (1970). Nucleic acid homologies among species of Saccharomyces. Journal of Bacteriology ror, 505-5I 2.

Brody, T. \& Williams, K. L. (I974). Cytological analysis of the parasexual cycle in Dictyostelium discoideum. Journal of General Microbiology 82, $37 \mathrm{I}-383$.

BYers, B. \& GoeTsCh, L. (I975). Electron microscopic observations on the meiotic karyotype of diploid and tetraploid Saccharomyces cerevisiae. Proceedings of the National Academy of Science of the United States of America 72, 5056-5060.

CAMPBell, I. (1972). Numerical analysis of the genera Saccharomyces and Kluyveromyces. Journal of General Microbiology 73, 279-301.

Fischer, P., Binder, M. \& WinTERSBERGER, U. (I975). A study of the chromosomes of the yeast Schizosaccharomyces pombe by light and electron microscopy. Experimental Cell Research 96, 1 5-22.

Franco, L., Johns, E. W. \& NavleT, J. M. (I974). Histones from baker's yeast. European Journal of Biochemistry 45, 83-89.

McCully, E. K. \& Robinow, C. F. (197I). Mitosis in the fission yeast Schizosaccharomyces pombe: a comparative study with light and electron microscopy. Journal of Cell Science 9, 475-507.

MORTIMER, R. K. \& HaWthoRne, D. C. (I969). The Yeasts, vol. I, pp. 386-460. Edited by A. H. Rose and J. S. Harrison. London and New York: Academic Press.

Robson, G. E. \& Williams, K. L. (I977). The mitotic chromosomes of the cellular slime mould Dictyostelium discoideum: a karyotype based on Giemsa banding. Journal of General Microbiology 99, I9I-200.

TAMAKI, H. (1965). Chromosome behaviour at meiosis in Saccharomyces cerevisiae. Journal of General Microbiology 41, 93-98.

Tingle, M., Herman, A. \& Halvorson, H. O. (1968). Characterisation and mapping of histidine genes in Saccharomyces lactis. Genetics 58, 361371 .

Wintersberger, U., Smith, P. \& Letnansky, K. (1973). Yeast chromatin. European Journal of Biochemistry 33, 123-I 30.

Wintersberger, U., BINDER, M. \& Fischer, P. (1975). Cytogenetic demonstration of mitotic chromosomes in the yeast Saccharomyces cerevisiae. Molecular and General Genetics 142, 13-17. 